\title{
Predicting disordered regions in proteins using the profiles of amino acid indices Pengfei Han ${ }^{1}$, Xiuzhen Zhang ${ }^{1}$ and Zhi-Ping Feng*2
}

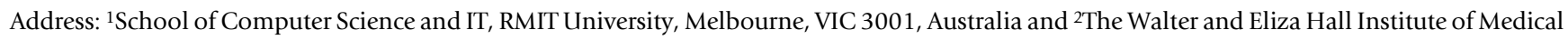
Research, Parkville, VIC 3050, Australia

Email: Pengfei Han - phan@cs.rmit.edu.au; Xiuzhen Zhang - xiuzhen.zhang@rmit.edu.au; Zhi-Ping Feng* - feng@wehi.edu.au

* Corresponding author

from The Seventh Asia Pacific Bioinformatics Conference (APBC 2009)

Beijing, China. 13-16 January 2009

Published: 30 January 2009

BMC Bioinformatics 2009, I0(Suppl I):S42 doi:I0.1186/I47I-2105-I0-SI-S42

This article is available from: http://www.biomedcentral.com/I47I-2/05/I0/SI/S42

(c) 2009 Han et al; licensee BioMed Central Ltd.

This is an open access article distributed under the terms of the Creative Commons Attribution License (http://creativecommons.org/licenses/by/2.0), which permits unrestricted use, distribution, and reproduction in any medium, provided the original work is properly cited.

\begin{abstract}
Background: Intrinsically unstructured or disordered proteins are common and functionally important. Prediction of disordered regions in proteins can provide useful information for understanding protein function and for high-throughput determination of protein structures.

Results: In this paper, algorithms are presented to predict long and short disordered regions in proteins, namely the long disordered region prediction algorithm DRaai- $L$ and the short disordered region prediction algorithm DRaai-S. These algorithms are developed based on the Random Forest machine learning model and the profiles of amino acid indices representing various physiochemical and biochemical properties of the 20 amino acids.

Conclusion: Experiments on DisProt3.6 and CASP7 demonstrate that some sets of the amino acid indices have strong association with the ordered and disordered status of residues. Our algorithms based on the profiles of these amino acid indices as input features to predict disordered regions in proteins outperform that based on amino acid composition and reduced amino acid composition, and also outperform many existing algorithms. Our studies suggest that the profiles of amino acid indices combined with the Random Forest learning model is an important complementary method for pinpointing disordered regions in proteins.
\end{abstract}

\section{Background}

Proteins are linear chains composed of 20 amino acids (aa), also called residues when they form chains by detaching water molecules, linked together by polypeptide bonds and folded into complex three-dimensional (3D) structures. Disordered regions (DRs) in protein sequence are structurally flexible and usually have low sequence complexity [1-4]. Physicochemically, DRs are enriched in charged or polar amino acids, and depleted in hydrophobic amino acids [5-7]. Proteins containing long DRs are called intrinsically unstructured or disordered proteins (IUPs or IDPs).

A number of protein disorder predictors have been developed by several groups, such as PONDR [8], RONN $[9,10]$, DisProt [11,12], NORSp [13,14], DISpro [15], DISOPRED and DISOPRED2 [16,17], DisEMBL [18], IUPred [19], DRIP-PRED [20] and Spritz [21], and more 
recently DisPSSMP [22], VSL1 and VSL2 [23,24], POODLE-L [25], POODLE-S [26], Ucon [27], PrDOS [28] and metaPrDOS [29]. Most existing predictors are based on the Neural Network and Support Vector Machine learning models. The features used to construct the prediction models include amino acid composition (AAC) or reduced amino acid composition (RAAC) combined with the physiochemical properties of amino acids including aromaticity, net charge, flexibility, hydropathy, coordination number and sequence complexity [8-10]. To achieve high prediction accuracy, typically algorithms use many features as input. Some algorithms are based on the sequence alignment scores from PSI-BLAST or protein secondary structure information $[16,17,21]$. Either approach lowers the efficiency of these algorithms and hinders their application in high-throughput analysis.

It has been shown that short disordered regions have different characteristics from long disordered regions [30]. Algorithms perform well in predicting long disordered regions rarely perform well in predicting short disordered regions. In this paper, algorithms for predicting short and long DRs are developed separately based on the Random Forest learning model [31] and the profiles of the amino acid indices. The algorithm for long disordered regions, DRaai- $\mathrm{L}$, can achieve an area of $85.1 \%$ under the receiver operating characteristic (ROC) curves in the 10 fold cross validation test. The algorithm targeting all kinds of disordered regions, DRaai-S, can achieve an area of $81.2 \%$ under the ROC curve in the 10 fold cross validation test and about $72.2 \%$ in the blind test on CASP7 targets. Both DRaai-L and DRaai-S achieve higher prediction accuracy as well as higher computation efficiency than many existing algorithms, which make them efficient tools for highthroughput prediction of disordered regions in proteins.

\section{Training and test data}

In this study, the training data is derived from DisProt (version 3.6) [32] and PDB-Select-25 (the Oct.2004 version) [33]. DisProt is a collection of disordered regions of proteins based on published literature descriptions. It has 472 proteins entries and 1121 disordered regions. Only long disordered regions (>30aa) in DisProt3.6 are used to train DRaai- $\mathrm{L}$, and it is denoted as DL-train hereafter. All disordered regions in DisProt3.6 were used to train DRaai-S, and it is denoted as DS-train hereafter. The ordered training data is extracted from PDB-Select-25, a representative set of protein data bank (PDB) chains that shows less than $25 \%$ sequence homology. We selected 366 high-resolution $(<2 \AA)$ segments with well-defined structures which has no missing backbone or side chain coordinates and contains at least 80 residues. This collection of ordered training set includes a total of 80324 residues, and is referred to as O-train hereafter. The CASP7 targets were used as an independent test dataset to blind test the performance of prediction. The disorder contents of CASP7 are very different from those of DisProt3.6. The CASP7 dataset contains 96 sequences with a total of 19,891 residues, where only 170 disordered regions, or $1,189(6 \%)$ residues are annotated as disordered. There is a significant amount (28\% in aa) of short disordered regions containing 1 or 2 aa, and only 4 are long DRs of $>30$ aa $(<2 \%$ in aa). While DisProt3.6 contains 352 regions of $>30$ aa with 47251 aa in total ( $36 \%$ in aa).

\section{The amino acid indices and feature selection}

The amino acid index (AA-index) database AAindex [34] is a database of numerical indices representing various physiochemical and biochemical properties of amino acids or pairs of amino acids. Especially the AAindex 1 database comprises 544 sets of numerical indices for the 20 amino acids, and all of them are derived from published literature.

The AA-indices that are highly correlated with the disordered or ordered status of the residues in the training protein sequences were used to construct the prediction model in our studies. The process of choosing these indices was implemented in three steps. First of all, given a set of indices and a training sequence, the training sequence is transformed into two vectors $\vec{V} 1$ and $\vec{V} 2 . \vec{V} 1$ is generated by replacing ordered and disordered resides with the number -1 and 1 respectively based on the annotations

from the databases. $\vec{V} 2$ is the result by substituting the amino acid code by the corresponding AA-index value.

Note that as different sets of AA-index are of different scales in the AA-index database, the Z-transformation $\left(P_{r}^{\prime}\right)$ is applied for each set of index before the substitution. For a set of AA-index, the $Z$-transformation is shown in Equation 1.

$$
P_{r}^{\prime}=\frac{P_{r}-\bar{P}}{\sigma}
$$

$P_{r}$ represents an AA-index value and $r$ varies for the 20 amino acids denoted as $1 . .20 . \bar{P}$ and $\sigma$ are the mean and standard deviation of the 20 AA-index values:

$$
\bar{P}=\frac{\sum_{r=1}^{20} P_{r}}{20}
$$

and

$$
\sigma=\sqrt{\frac{1}{20} \sum_{r=1}^{20}\left(P_{r}-\bar{P}\right)^{2}}
$$


After the AA-index substitution, the structural influence to a residue by its surroundings is calculated using a smooth function. The Savitzky-Golay filter [35] is used to smooth

both $\vec{V} 1$ and $\vec{V} 2$ in our study with a window of 17 aa. This filter essentially performs a polynomial regression on the $\vec{V} 1$ and $\vec{V} 2$ to determine the smoothed value for each point. The main advantage of Savitzky-Golay is to preserve features of the distribution such as relative max score, min score and width of disordered or ordered regions, which are usually "flattened" by other smooth techniques. The smoothed vectors $\vec{V} 1^{\prime}$ and $\vec{V} 2^{\prime}$ denote the results of filtering $\vec{V} 1$ and $\vec{V} 2$ respectively.

Finally the correlation coefficient of an AA-index set and a protein sequence is calculated as shown in Equation 4, where $N$ represents the length of the sequence under consideration.

$$
R_{\vec{V} 1^{\prime} \vec{V} 2^{\prime}}=\frac{\sum_{r=1}^{N}\left(\vec{V} 1^{\prime}-\overline{\vec{V}} 1^{\prime}\right)\left(\vec{V} 2^{\prime}-\overline{\vec{V}} 2^{\prime}\right)}{(N-1) \sigma_{\vec{V} 1}, \sigma \vec{V} 2^{\prime}}
$$

The correlation coefficient $R_{\vec{V} 1^{\prime} \vec{V} 2^{\prime}}$ is in the range [-1..1]. A positive coefficient indicates that the set of AA-indices is positively correlated with the order/disorder status of residues in the sequence, whereas a negative coefficient indicates negative correlation.

The sets of AA-indices that are mostly related to the disorder/order status of residues in all our training sequences were used to construct the prediction model. Specifically these sets of indices were chosen so that

- To maximize the summarization of the absolute correlation coefficients of the index over all training sequences.

- To maximize the number of protein sequences that the index uniformly correlates with.

Based on the above two criteria, the top 40 AA-index sets were selected. Among the 40 sets, many are highly correlated (with correlation coefficient of at least 0.8 ), and as a result five representative index sets were selected, as shown in Table 1.

From the description of these 5 sets of AA-indices listed in Table 1, we can see that they are strongly correlated with protein structures. For example, index BULH740101 represents hydrophobicity while it is known that ordered regions tend to be hydrophobic, indices CHOP780203 and CHOP780211 represent alpha and turn propensities which has been widely used in secondary structure prediction.
The Moreau-Broto autocorrelation functions of AA-indices The profiles of amino acid indices along a protein sequence have been used in the protein structural and functional classification studies [36-38]. Given an AAindex set, the normalized Moreau-Broto autocorrelation coefficient for an amino acid protein sequence is defined in Equation 5:

$$
A C(d)=\frac{1}{N-d} \times \sum_{i=1}^{N-d} P_{i} P_{i+d}
$$

where $N$ is the length of the sequence under consideration, and $d$ is an integer larger than zero and describe the lag of the autocorrelation or the distance in the number of residues separated in the protein sequence. In this study, $d$ is set to $1 . .30 . P_{i}$ and $P_{i+d}$ are the AA-index values at positions $i$ and $i+d$ normalized by Z-transformation respectively. We used the Moreau-Broto autocorrelation functions generated from smoothed vector $\vec{V} 2^{\prime}$ under different windows as input to develop the DRaai- $\mathrm{L}$ algorithm, and used the vector $R_{\vec{V} 1^{\prime} \vec{V} 2^{\prime}}=\frac{\sum_{r=1}^{N}\left(\vec{V} 1^{\prime}-\overline{\vec{V}} 1^{\prime}\right)\left(\vec{V} 2^{\prime}-\overline{\vec{V}} 2^{\prime}\right)}{(N-1) \sigma \vec{V} 1^{\prime}, \sigma \vec{V} 2^{\prime}}$ directly to develop the DRaai-S algorithm.

\section{Methods}

The Random Forest machine learning model is the underlying model in this study. A random forest is an ensemble of unpruned decision trees, where each tree is grown using a (bootstrap) subset of the training dataset [39]. Bootstrap is the training set drawn randomly from the original training set with an equal number of training samples. Each tree induced from bootstrap samples grows to full length and the number of trees in the forest is adjustable. After training, every path from the root of a tree to a leaf gives one if-then rule and can be used for prediction. As an ensemble machine learning model the random forest has no risk of overfitting with an increasing number of trees. However, after certain point, the increase of number of trees leads to trivial improvement of prediction accuracy while prolonging the time of training and prediction significantly. The random forest implementation of the WEKA data mining package [40] is used to build our models.

\section{DRaai-L: predicting long DRs using AA-indices}

DL-train and O-train are used to train the algorithm DRaai-L. For each ordered or disordered region in the $D L$ train and O-train datasets, a window of $w$ aa (by default $w$ $=31$ ) slides along a sequence from $\mathrm{N}$-terminus to C-terminus one residue at a time. The Moreau-Broto autocorrelation of the 5 sets of AA-indices in each window is calculated with $d$ assigned from $1 . .30$. So $n=5 \times 30=150$ 
Table I: Amino acid indices related to (dis)order. The five sets of amino acid indices that are most correlated to the (dis)order of proteins are the features used in prediction.

\begin{tabular}{ll}
\hline AA-index set & Description \\
\hline VINM940I02 & Normalized flexibility parameters (B-values) for each residue surrounded by none rigid neighbours \\
BULH740101 & Surface tension of amino acid solutions: A hydrophobicity scale of the amino acid residues \\
PUNT030I02 & Knowledge-based membrane-propensity scale from 3D_Helix in MP- topo databases \\
CHOP780203 & Normalized frequency of beta-turn \\
CHOP7802II & Normalized frequency of C-terminal non beta region
\end{tabular}

elements are generated from a window. When a window of $w$ residues slides along a protein sequence of $L_{i}$ residues, the sequence is represented by $\left(L_{i} / w\right) \times n$ elements. These elements are used as the input parameters to the random forest to train the DRaai-L model.

For a query sequence, a window slides along the sequence and its corresponding vectors $\vec{V} 2$ ' is computed using the Moreau-Broto autocorrelation functions. The smoothed vectors $\vec{V} 2$ ' are then input to the DRaai-L model, and the disordered/ordered status of each residue is predicted.

\section{DRaai-S: predicting short DRs using AA-indices}

All disordered regions in DisProt3.6, DS-train, were used to train DRaai-S. Each amino acid sequence in the training set was replaced with numerical sequences by the 5 sets of AA-indices and smoothed using the Savitzky-Golay filter (with a window of $17 \mathrm{aa}$ ). Then the smoothed vectors $\vec{V} 2^{\prime}$ are directly used as input parameters to develop the DRaai-S model,

To predict the disorder of a query sequence, the sequence is transformed similarly to the 5 smoothed vectors $\vec{V} 2$ ', and then they are input to the DRaai-S model to predict the disorder/order of each residue.

\section{Evaluation}

The distribution of ordered/disordered residues are very imbalanced in both DisProt3.6 and CASP7. With the fact that disordered residues are by far the minority in both databases, overall accuracy (Q2) is not a good measure to evaluate disorder prediction algorithms [41]. Ideally a disorder algorithm should be highly sensitive on disordered regions while not producing many false positive predictions. The confusion matrix of an algorithm, which comprises True Positive (TP), False Positive (FP), True Negative (TN) and False Negative (FN), can be used to evaluate the performance of the algorithm. Note that in the context of disorder prediction $\mathrm{P}$ and $\mathrm{N}$ are the total number of labelled disordered and ordered residues respectively.
The receiver operating characteristic (ROC) curves were used to evaluate the prediction accuracy. Each point of a ROC curve is defined by a pair of values for the false positive rate $(x=F P / N)$ and the true positive rate $(y=T P / P)$. For a prediction algorithm, by adjusting the parameters, the true positive rate can be plotted under different false positive rates and a smooth ROC curve can be obtained.

The performance of DRaai- $\mathrm{L}$ and DRaai-S is measured in different methods as described below.

- The Sensitivity is the true positive rate, which is the percentage of residues correctly predicted as disordered in relation to the total number of actual disordered residues.

- The Precision is the percentage of true positives in relation to the total number of predicted positives.

- The Specificity is the percentage of residues correctly predicted as ordered in relation to the total number of ordered residues. The false positive rate is 1 -Specificity.

- $S_{\text {product }}$ is a single measurement combining sensitivity and specificity: $S_{\text {product }}=$ Sensitivity $\times$ specificity. $S_{\text {product }}$ favours disorder prediction.

- The Matthew Correlation Coefficient (MCC) ranges between -1 and +1 , and favors correct predictions of disordered residues. MCC is defined as

$$
\frac{T P \times T N-F P \times F N}{\sqrt{(T P+F P) \times(T P+F N) \times(T N+F P) \times(T N+F N)}} .
$$

- $S_{w}$ is a measurement that assigns class weights that are reversely related to class distribution. As a result, $S_{w}$ rewards models for correctly predicting a disordered residue. $S_{w}$ was used in assessing the prediction of disordered residues in CASP6 and CASP7. $S_{w}$ is defined as

$$
\frac{W_{\text {disorder }} \times T P-W_{\text {order }} \times F P+W_{\text {order }} \times T N-W_{\text {disorder }} \times F N}{W_{\text {disorder }} \times P+W_{\text {order }} \times N},
$$

where $W_{\text {disorder }}$ and $W_{\text {order }}$ are the weights for disorder and order respectively. $W_{\text {disorder }}$ and $W_{\text {order }}$ should be set to be 
inversely proportional to the disorder and order content in the data under consideration. For evaluation on DisProt3.6, $W_{\text {disorder }}=85$ and $W_{\text {order }}=15$. For evaluation on on CASP7, $W_{\text {disorder }}=94$ and $W_{\text {order }}=6$.

The random forest package we use provides the out-of-bag test to estimate prediction error rate using data randomly withheld from each iteration of tree development. However this approach significantly overestimates the performance when a window technique is used.

The performance of both DRaai-L and DRaai-S are evaluated on DisProt3.6 using 10-fold cross validation. The performance of DRaai-S is further evaluated by blind test on CASP7 targets.

DRaai-L and DRaai-S are compared with algorithms based on the Random Forest model but constructed using the amino acid composition (AAC) and reduced AAC (RAAC) [42] information of the primary sequences. They are also compared with other existing disorder prediction algorithms.

\section{Results}

The results of evaluating DRaai-L and DRaai-S using 10fold cross validation tests on DisProt3.6 and blind test on CASP7 are presented separately.

\section{The performance of DRaai-L}

The performances of DRaai- $\mathrm{L}$ under different number of trees for the random forest model and different $d$ values for the Moreau-Broto autocorrelation coefficients are presented using ROC curves shown in Figure 1. The area under the ROC for the model trained with 50 trees and the auto-correlation coefficients generated from $d=1,2,30$ aa is $85.1 \%$. Even for the model trained with 10 trees and the auto-correlation coefficients generated from $d=1$, $2, \ldots, 15$ aa, the area under the ROC can reach $82.7 \%$. This result is better than that trained with AAC $(78.6 \%$, under 50 trees and $d=1,2, \ldots, 30)$ or RAAC $(74.1 \%$, under 10 trees and $d=1,2, \ldots, 15)$. This result is also better than that of most other available algorithms, as indicated by the separate points in Figure 1.

Table 2 describes the performance of DRaai-L in comparison with other published algorithms. The performance is measured in terms of Sensitivity, Precision, Specificity, $S_{\text {product }}$, MCC and $S_{w}$. DRaai-L is with a setting of 50 trees and $d=1,2, \ldots, 30$. With these six methods of evaluation, the performance of DRaai-L is just below IUPred, but better than most other predictors.

\section{The performance of DRaai-S}

Figure 2 shows the ROC curves for DRaai-S under 10 fold cross validation and on CASP7 targets. The area under
ROC of DRaai-S in 10 fold cross validation is $81.2 \%$, while it dropped to $72.2 \%$ when used to predict the CASP7 targets. Table 3 describes the performance of DRaai-S on CASP7 in comparison with other predictors. DRaai-S is with a setting of 10 trees and a smoothing window of 17 aa. The results in both Figure 2 and Table 3 demonstrate that DRaai-S can achieve comparable or even more accurate prediction than some published algorithms.

In summary, by using the simple AA-index information, both DRaai-L and DRaai-S have shown better performance than many well developed published algorithms. DRaai-L and DRaai-S have the potential to be further improved by adjusting the sets of AA-indices, the number of residues to be smoothed, and the number of residues considered in the auto-correlation function.

\section{Discussion}

The good performance of DRaai-L compared with the other published algorithms shown in Figure 1 and Table 2 indicates that the continuous correlations among the nearby residues along a primary sequence implies ordered/disordered structural information. It is well known that the residues involved in ordered structures are always close to other residues in space. In other words, they are constrained by backbone or side chain interactions from other residues, and hence they have higher density in the contact map [27]. Indeed the auto-correlation functions used in DRaai-L reflect such contact information. If the residues in a fragment of more than 30 aa do not show any kind of correlation between each other,

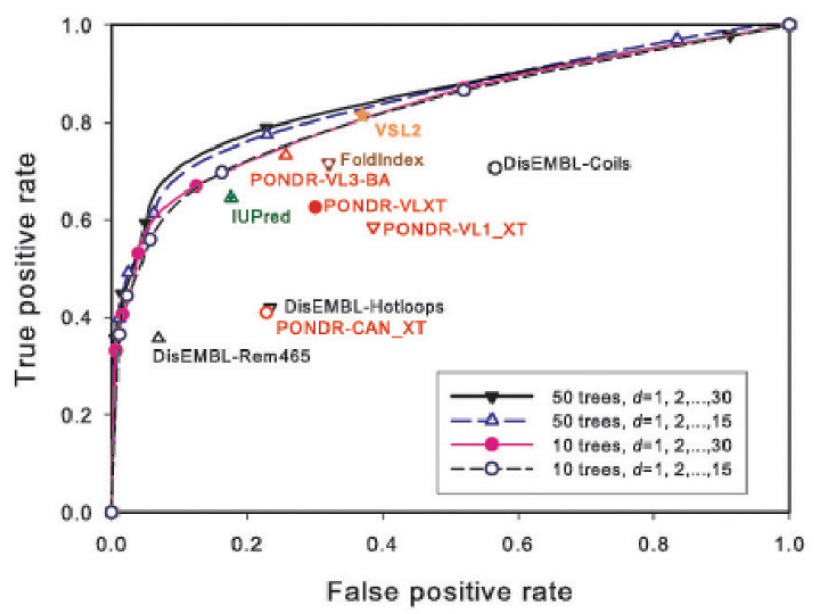

Figure I

Performance of DRaai-L. The ROC curves of DRaai-L in 10 -fold cross validation test. All independent points in the figure are results obtained from the respective online predictors with their default settings. 
Table 2: The performance DRaai-L on DisProt3.6. The performance of DRaai- $L$ in the independent test on $10 \%$ of DisProt3.6 targets under various measures in comparison with other predictors.

\begin{tabular}{|c|c|c|c|c|c|c|}
\hline Algorithm & Sensitivity & Precision & Specificity & $S_{\text {product }}$ & MCC & $S_{w}$ \\
\hline DisEMBL(Coil) & 0.71 & 0.33 & 0.43 & 0.31 & 0.13 & 0.24 \\
\hline DisEMBL(Rem465) & 0.36 & 0.67 & 0.93 & 0.33 & 0.36 & 0.29 \\
\hline DisEMBL(Hot Loop) & 0.42 & 0.41 & 0.77 & 0.32 & 0.18 & 0.19 \\
\hline Foldlndex & 0.72 & 0.46 & 0.68 & 0.49 & 0.36 & 0.40 \\
\hline IUPred & 0.65 & 0.59 & 0.82 & 0.53 & 0.46 & 0.47 \\
\hline PONDR(CANXT) & 0.41 & $0.4 I$ & 0.77 & 0.32 & 0.18 & 0.18 \\
\hline PONDR(VL) & 0.55 & 0.55 & 0.77 & 0.42 & 0.32 & 0.29 \\
\hline PONDR(VLXT) & 0.63 & 0.45 & 0.70 & 0.44 & 0.30 & 0.33 \\
\hline PONDR(XL) & 0.59 & 0.37 & 0.61 & 0.36 & 0.18 & 0.20 \\
\hline VSL2 & 0.76 & 0.79 & 0.79 & 0.60 & 0.55 & 0.55 \\
\hline DRaai-L & 0.78 & 0.80 & 0.80 & 0.62 & 0.58 & 0.57 \\
\hline
\end{tabular}

it is very unlikely that these residues are constrained by each other or form stable contacts, they therefore have high propensity to be disordered.

The prediction results of DRaai-S on DisProt3.6 and CASP7 shown in Figure 2 and Table 3 indicate that the position specific profiles of the physiochemical properties of residues determine whether they are involved in short disordered regions. The poor performance of DRaai-S compared with DRaai-L indicates that accurately predicting short disordered regions is significantly more challenging than predicting long disordered regions. This is partially due to the difficulty of extracting local sequence information, but more importantly due to the lack of sufficient robust short disordered regions in the training dataset. Therefore, a short DR predictor trained from very limited number of short disordered regions can produce a high false positive rate or fluctuated prediction accuracy.

CASP targets are a typical set of highly ordered globular proteins that are suitable for protein structural determination by either NMR or X-crystallography. As such the distribution of disorder in CASP targets is not a typical representation of disorder in all proteomes. Indeed the distribution of short DRs in DisProt3.6 is significantly different. Among the limited number of disordered regions in CASP targets, the majority are either very short or distributed in the terminal regions. However protein sequence-structural relationship in the terminal regions has not been well established [43]. As a result the disordered regions in CASP targets are extremely difficult to predict. To improve the prediction accuracy on CASP targets, many existing prediction algorithms use various features including predicted secondary structure and position specific scoring matrix, which typically requires lengthy PSI-BLAST search. DRaai-S uses the simple and uniform AA-index information and can efficiently predict disordered regions in CASP targets, with a reasonable accuracy that has a great promise to be further improved.

\section{Conclusion}

Protein disorder studies are becoming increasingly important because IUPs are common and functionally important. Experimental studies of IUPs are expensive and time consuming. In this paper we have presented two algorithms DRaai-L and DRaai-S for predicting disordered regions in proteins, using the profiles of AA-indices and the Random Forest machine learning model. By using Moreau-Broto auto-correlation functions and profiles of AA-indices and Savitzky-Golay filter, long disordered regions and short disordered regions can be accurately predicted with DRaai-L and DRaai-S respectively.

With the simple and uniform AA-index information, both DRaai-L and DRaai-S outperform some well developed

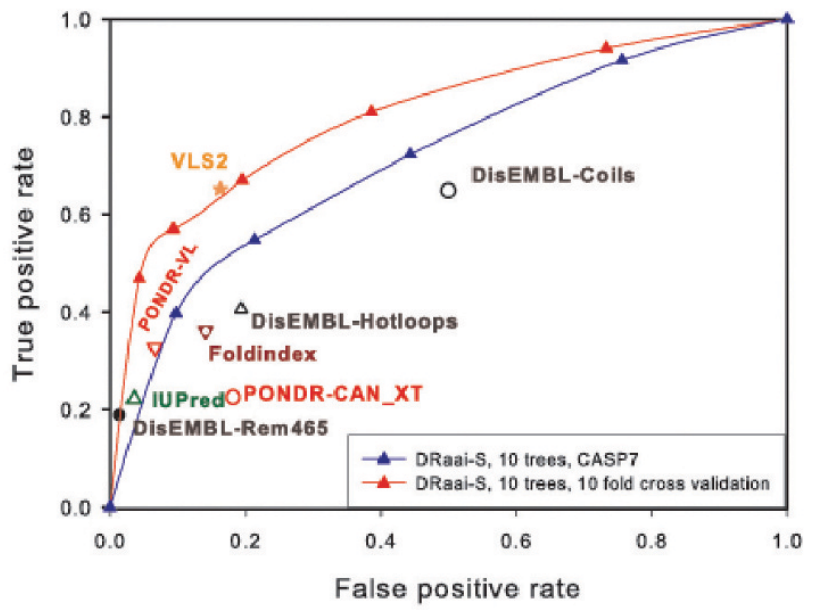

\section{Figure 2}

Performance of DRaai-S. The ROC curves of DRaai-S in I0-fold cross validation test and blind test on CASP7. All independent points in the figure are results on CASP7 targets obtained from the respective online predictors with their default settings. 
Table 3: The performance DRaai-S on CASP7. The performance of DRaai-S of independent test on CASP7 targets under various measures in comparison with other predictors.

\begin{tabular}{|c|c|c|c|c|c|c|}
\hline Algorithm & Sensitivity & Precision & Specificity & Sproduct & MCC & Sw \\
\hline DisEMBL(Coil) & 0.65 & 0.08 & 0.50 & 0.33 & 0.07 & 0.15 \\
\hline DisEMBL(Rem465) & 0.19 & 0.47 & 0.99 & 0.19 & 0.27 & 0.18 \\
\hline DisEMBL(Hot Loop) & 0.41 & 0.12 & 0.81 & 0.33 & 0.12 & 0.21 \\
\hline Foldlndex & 0.36 & 0.14 & 0.86 & 0.31 & 0.14 & 0.22 \\
\hline IUPred & 0.22 & 0.28 & 0.96 & 0.21 & 0.21 & 0.19 \\
\hline PONDR(CANXT) & 0.23 & 0.07 & 0.82 & 0.18 & 0.03 & 0.05 \\
\hline PONDR(VL) & 0.33 & 0.24 & 0.93 & 0.30 & 0.23 & 0.26 \\
\hline PONDR(VLXT) & 0.46 & 0.12 & 0.79 & 0.36 & 0.14 & 0.25 \\
\hline PONDR(XL) & 0.30 & 0.06 & 0.72 & 0.22 & 0.01 & 0.02 \\
\hline VSL2 & 0.73 & 0.21 & 0.85 & 0.61 & 0.33 & 0.58 \\
\hline DRaai-S & 0.55 & 0.14 & 0.79 & 0.43 & 0.19 & 0.34 \\
\hline
\end{tabular}

algorithms, with high computing efficiency. This makes them competitive tools to be used in large-scale structural analyses and in comparative proteome studies.

\section{List of abbreviations used}

aa: amino acid; AAC: amino acid composition; AA-index: amino acid index; DR: disordered region; IDP: intrinsically disordered protein; IUP: intrinsically unstructured protein; RAAC: reduced amino acid composition; ROC: receiver operating characteristic.

\section{Competing interests}

PH is supported by an Australian Postgraduate Award. XZ is supported in part by an RMIT Emerging Researcher Grant. ZPF is supported by an APD Award from the Australian Research Council.

\section{Authors' contributions}

PH carried out the algorithm implementation and performance evaluation. XZ and ZPF participated in the design of the study, and drafted the manuscript.

\section{Acknowledgements}

The authors thank Dr Marc Cortese for his explanation of the DisProt database.

This article has been published as part of BMC Bioinformatics Volume I0 Supplement I, 2009: Proceedings of The Seventh Asia Pacific Bioinformatics Conference (APBC) 2009. The full contents of the supplement are available online at http://www.biomedcentral.com/I47/-2105//0?issue=SI

\section{References}

I. Ward JJ, Sodhi JS, McGuffin LJ, Buxton BF, Jones DT: Prediction and functional analysis of native disorder in proteins from the three kingdoms of life. J Mol Biol 2004, 337:635-645.

2. Romero P, Obradovic Z, Li X, Garner EC, Brown C], Dunker AK Sequence complexity of disordered protein. Proteins 200I, 42:38-48.

3. Coeytaux K, Poupon A: Prediction of unfolded segments in a protein sequence based on amino acid composition. Bioinformatics 2005, $21: 1891-1900$.
4. Radivojac P, Obradovic Z, Brown Cl, Dunker AK: Prediction of boundaries between intrinsically ordered and disordered protein regions. Pac Symp Biocomput 2003:216-227.

5. Weathers EA, Paulaitis ME, Woolf TB, Hoh JH: Reduced amino acid alphabet is sufficient to accurately recognize intrinsically disordered protein. FEBS Lett 2004, 576:348-352.

6. Hansen JC, Lu X, Ross ED, Woody RW: Intrinsic protein disorder, amino acid composition, and histone terminal domains. | Biol Chem 2006, 28 I: | 853-1856.

7. Uversky VN, Oldfield CJ, Dunker AK: Showing your ID. J Mol Recognit 2005, 18:343-84.

8. Li X, Romero P, Rani M, Dunker AK, Obradovic Z: Predicting protein disorder for $\mathrm{N}-, \mathrm{C}_{-}$, and internal regions. Genome Informatics 1999, 10:30-40.

9. Thomson R, Esnouf R: Prediction of natively disordered regions in proteins using a bio-basis function neural network. LNCS 3177 2004:108-116.

10. Yang ZR, Thomson R, McNeil P, Esnouf RM: RONN: the bio-basis function neural network technique applied to the detection of natively disordered regions in proteins. Bioinformatics 2005 , 21(16):3369-3376

II. Obradovic Z, Peng K, Vucetic S, Radivojac P, Brown C, Dunker AK: Predicting intrinsic disorder from amino acid sequence. Proteins 2003, 53(Suppl 6):566-572.

12. Peng K, Vucetic S, Radivojac P, Brown C], Dunker AK, Obradovic Z: Optimizing Intrinsic Disorder Predictors with Protein Evolutionary Information. J Bioinform Comp Biol 2005, 3(I):35-60.

13. Liu J, Tan $H$, Rost $B$ : Loopy proteins appear conserved in evolution. J Mol Biol 2002, 322:53-64.

14. Liu J, Rost B: NORSp: predictions of long regions without regular secondary structure. Nucleic Acids Res 2003, 31:3833-3835.

15. Cheng J, Sweredoski M, Baldi P: Accurate prediction of protein disordered regions by mining protein structure data. Dato Mining and Knowledge Discovery 2005:213-222.

16. Ward JJ, McGuffin LJ, Bryson K, Buxton BF, Jones DT: The DISOPRED server for the prediction of protein disorder. Bioinformatics 2004, 20:2138-2/39.

17. Jones DT, Ward J]: Prediction of disordered regions in proteins from position specific score matrices. Proteins 2003, 53:573-578

18. Linding R, Jensen LJ, Diella F, Bork P, Gibson TJ, Russell RB: Protein disorder prediction: implications for structural proteomics. Structure 2003, II: 1453-1459.

19. Dosztanyi Z, Csizmok V, Tompa P, Simon I: The Pairwise Energy Content Estimated from Amino Acid Composition Discriminates between Folded and Intrinsically Unstructured Proteins. J Mol Biol 2005, 347:827-839.

20. Order/Disorder Prediction for Protein Sequences [http:// www.sbc.su.se/ maccallr/disorder/]

21. Vullo A, Bortolami O, Pollastri G, Tosatto SCE: Spritz: a server for the prediction of intrinsically disordered regions in protein sequences using kernel machines. Nucleic Acids Res 2006, 34:. 
22. Su CT, Chen CY, Ou YY: Protein disorder prediction by condensed PSSM considering propensity for order or disorder. BMC Bioinformatics 2006, 7:319.

23. Obradovic Z, Peng K, Vucetic S, Radivojac P, Dunker AK: Exploiting Heterogeneous Sequence Properties Improves Prediction of Protein Disorder. Proteins 2005, 6 I (Suppl 7): I76-I82.

24. Peng K, Radivojac P, Vucetic S, Dunker AK, Obradovic Z: Lengthdependent prediction of protein intrinsic disorder. $B M C$ Bioinformatics 2006, 7:208

25. Hirose S, Shimizu K, S K, Y K, T N: POODLE-L: a two-level SVM prediction system for reliably predicting long disordered regions. Bioinformatics 2007, 23(16):2046-53.

26. Shimizu Kea: POODLE-S: Web application for predicting protein disorder by using physiochemical features and reduced amino acid set of a position-specific scoring matrix. Bioinformatics 2007, 23(16):2337-38.

27. Schlessinger A, Punta $M$, Rost $B$ : Natively unstructured regions in proteins identified from contact predictions. Bioinformatics 2007, 23:2376-2384

28. Ishida T, Kinoshita K: PrDOS: prediction of disordered protein regions from amino acid sequence. Nucleic Acids Research 2007 35:W460-W464.

29. Ishida T, Kinoshita K: Prediction of disordered regions in proteins based on the meta approach. Bioinformatics 2008, 24:1344-I348.

30. Peng K, Vucetic S, Radivojac P, Brown C, Dunker A, Obradovic Z: Optimizing Long Intrinsic Disorder Predictors with Protein Evolutionary Information. J Bioinform Comp Biol 2005, 3:35-60.

31. Breiman L: Random Forests. Machine Learning 200I, 45:5-32

32. Vucetic S, Obradovic Z, Vacic V, Radivojac P, Peng K, lakoucheva LM, Cortese MS, Lawson JD, Brown CJ, GSikes J, Newton CD, Dunker AK: DisProt: A Database of Protein Disorder. Bioinformatics 2005, $21: 137-140$

33. Hobohm U, Sander C: Enlarged representative set of protein structures. Protein Sci 1994, 3:522.

34. Kawashima S, Pokarowski P, Pokarowska M, Kolinski A, Katayama T, Kanehisa M: AAindex: amino acid index database, progress report 2008. Nucleic Acids Research 2008, 36:D202-D205.

35. Schreiber T, Schmitz A: Surrogate time series. Physica 2000, DI 42:346.

36. Dubchak I, Muchnik I, Mayor C, Dralyuk I, Kim S: Recognition of a protein fold in the context of the Structural Classification of Proteins (SCOP) classification. Proteins 1999, 35:40 I-407.

37. Feng ZP, Zhang CT: Prediction of membrane protein types based on the hydrophobic index of amino acids. J Protein Chem 2000, 19:269-275

38. Cai CZ, Han LY, Ji ZL, Chen YZ: Enzyme family classification by support vector machines. Proteins 2004, 55:66-76.

39. Breiman L: Random Forests Technical Report for Version 3. 2001.

40. Witten I, Frank E: Data Mining: Practical Machine Learning Tools and Techniques 2nd edition. 2005 [http://www.cs.waikato.ac.nz/ml/weka/] Morgan Kaufmann Publishers

4I. Jin $Y$, Dunbrack RLJ: Assessment of disorder predictions in CASP6. Proteins 2005, 61:167-175.

42. Han P, Zhang X, Norton R, Feng ZP: Predicting disordered regions in proteins based on decision trees of reduced amino acid composition. J Comput Biol 2006, 13(10): I723-1734.

43. Ferron F: A Practical Overview of Protein Disorder Prediction Methods. Proteins 2006, 65: I-I4.
Publish with Bio Med Central and every scientist can read your work free of charge

"BioMed Central will be the most significant development for disseminating the results of biomedical research in our lifetime. "

Sir Paul Nurse, Cancer Research UK

Your research papers will be:

- available free of charge to the entire biomedical community

- peer reviewed and published immediately upon acceptance

- cited in PubMed and archived on PubMed Central

- yours - you keep the copyright

Submit your manuscript here:

http://www.biomedcentral.com/info/publishing_adv.asp
BioMedcentral 